UMDGR-95-019

gr-qc/9410023

\title{
Topology of Event Horizons and Topological Censorship
}

\author{
Ted Jacobson円 and Shankar Venkataramanif \\ Dept. of Physics, Univ. of Maryland, College Park, MD 20742-4111
}

\begin{abstract}
We prove that, under certain conditions, the topology of the event horizon of a four dimensional asymptotically flat black hole spacetime must be a 2 -sphere. No stationarity assumption is made. However, in order for the theorem to apply, the horizon topology must be unchanging for long enough to admit a certain kind of cross section. We expect this condition is generically satisfied if the topology is unchanging for much longer than the light-crossing time of the black hole.

More precisely, let $M$ be a four dimensional asymptotically flat spacetime satisfying the averaged null energy condition, and suppose that the domain of outer communication $\mathcal{C}_{K}$ to the future of a cut $K$ of $\mathcal{I}^{-}$is globally hyperbolic. Suppose further that a Cauchy surface $\Sigma$ for $\mathcal{C}_{K}$ is a topological 3-manifold with compact boundary $\partial \Sigma$ in $M$, and $\Sigma^{\prime}$ is a compact submanifold of $\bar{\Sigma}$ with spherical boundary in $\Sigma$ (and possibly other boundary components in $M / \Sigma$ ). Then we prove that the homology group $H_{1}\left(\Sigma^{\prime}, Z\right)$ must be finite. This implies that either $\partial \Sigma^{\prime}$ consists of a disjoint union of 2-spheres, or $\Sigma^{\prime}$ is nonorientable and $\partial \Sigma^{\prime}$ contains a projective plane. Further, $\partial \Sigma=\partial I^{+}[K] \cap \partial I^{-}\left[\mathcal{I}^{+}\right]$, and $\partial \Sigma$ will be a cross section of the horizon as long as no generator of $\partial I^{+}[K]$ becomes a generator of $\partial I^{-}\left[\mathcal{I}^{+}\right]$. In this case, if $\Sigma$ is orientable, the horizon cross section must consist of a disjoint union of 2 -spheres.
\end{abstract}

\footnotetext{
1jacobson@umdhep.umd.edu

${ }^{2}$ cat@wam.umd.edu
} 
Hawking proved that each connected component of the event horizon of a stationary black hole in four dimensional general relativity has the topology of a 2-sphere [四]. His method of proof was to show that if the horizon had any other topology, and if the dominant energy condition is satisfied, one could always find a (possibly marginally) outer-trapped 2-surface outside the horizon. This would then imply failure of either the null convergence condition or cosmic censorship, since these imply that such a trapped surface must lie inside an event horizon.

Hawking's theorem was later generalized by Gannon[2] who proved, replacing stationarity by some weaker assumptions, that the horizon must be either spherical or toroidal. Gannon's assumptions include the dominant energy condition and the assumption that the horizon is smooth to the future of some slice, which entails in particular that no new generators enter to the future of that slice. Quite recently Browdy and Galloway [3] have proved, also without the stationarity assumption, that a cross section of a horizon must be spherical. Their assumptions include a null energy condition and the assumption that no new generators enter the horizon on or (therefore) to the future of the cross section. They also prove that the region of a partial Cauchy surface outside the black hole and inside a certain "well-behaved" sphere is compact and simply connected.

Making use of the topological censorship theorem of Friedman, Schleich, and Witt [1], we prove in this paper that a cross section of the event horizon must be topologically a 2-sphere, provided the horizon topology persists for long enough in a sense that will be made precise below. In the process we obtain an explicit restriction on the spatial topology of the domain of outer communication. A somewhat similar argument for the case of stationary black holes has been given by Chruściel and Wald [5].

The basic idea behind our theorem is that topological censorship forbids a nonsimply connected event horizon since a causal curve from $\mathcal{I}^{-}$to $\mathcal{I}^{+}$can be linked with such a horizon and not deformable to infinity within the domain of outer communication. More precisely, the idea is the following. Let $\mathcal{M}$ be an asymptotically flat spacetime and let $K$ be a cut of $\mathcal{I}^{-}$. We define the domain of outer communication to the future of $K$ by $\mathcal{C}_{K}:=I^{+}[K] \cap I^{-}\left[\mathcal{I}^{+}\right]$. Suppose $\mathcal{C}_{K}$ is globally hyperbolic, and that the averaged null energy condition holds in $\mathcal{C}_{K}$. The topological censorship theorem applied to the spacetime $\mathcal{C}_{K}$ then implies that all causal curves from $\mathcal{I}^{-}$to $\mathcal{I}^{+}$in $\mathcal{C}_{K}$ must be deformable to a simply connected neighborhood of infinity. [Otherwise, according to the 
argument of [4], $\mathcal{C}_{K}$ would not be simply connected, and there would be a surface that is outer trapped with respect to, and visible from, one of the copies of $\mathcal{I}^{+}$in the covering space of $\mathcal{C}_{K}$. The existence of such a trapped surface would be inconsistent with the assumed properties of $\mathcal{C}_{K}$.]

Having assumed that $\mathcal{C}_{K}$ is globally hyperbolic, it follows that it is topologically $\Sigma \times R$, where $\Sigma$ is any Cauchy surface[6]. Choosing an arbitrary nowhere vanishing timelike vector field $t^{a}$ on $\mathcal{C}_{K}$, we can identify all leaves of the foliation with one reference leaf, by flowing along the integral curves of $t^{a}$. Thus we can represent $\mathcal{C}_{K}$ as the product set $\Sigma \times R$.

It seems reasonable at first to expect that topological censorship would imply that $\Sigma$ is simply connected. If $\Sigma$ were not simply connected, there would be a noncontractible closed curve $\lambda$ in $\Sigma$ based at infinity. One could attempt to "lift" $\lambda$ to a timelike curve from $\mathcal{I}^{-}$to $\mathcal{I}^{+}$, that is not deformable to a neighborhood of infinity, by breaking $\lambda$ at infinity and pushing one end off to the future and the other end off to the past. [We are assuming here that $\Sigma$ has three or more dimensions since in two dimensions one can have a noncontractible curve that is nevertheless deformable to a neighborhood of infinity.] However, in order for such a strategy to work, it is necesary that one doesn't "run out of time", and there seems to be no guarantee that this cannot happen. For example, if a new horizon forms, some integral curve(s) of $t^{a}$ might necessarily have finite duration. Or, there might be an inflating region in the spacetime across which $\lambda$ could not be causally lifted. Thus we have not succeeded in showing that $\Sigma$ is simply connected. [If the spacetime is assumed to be stationary, these obstructions do not occur, and one can prove that $\Sigma$ is simply connected, as shown in [5].]

Since the above strategy for proving that $\Sigma$ must be simply connected meets with obstacles we were forced to come up with a different strategy. The one we found involves the first homology group with integer coefficients $H_{1}(\Sigma, Z)$, rather than the fundamental group $\pi_{1}(\Sigma)$, and it is applicable only in the case of $3+1$ spacetime dimensions. $\left[H_{1}(\Sigma)\right.$ is equivalent to the abelianization of the fundamental group $\pi_{1}(\Sigma)$ of $\Sigma$, i.e., the quotient of $\pi_{1}(\Sigma)$ by its commutator subgroup. (Here and hereafter we suppress explicit reference to the ring of integers $Z$ in the notation for the homology group.)]

In order to employ the topological results of [7] we must work with compact topological manifolds with boundary. Our theorem will refer to a compact submanifold $\Sigma^{\prime} \subset \bar{\Sigma}$ of the closure of $\Sigma$ with a single 2 -spherical boundary $S$ in $\Sigma$. In general $\Sigma^{\prime}$ will also possess boundary components in $\partial \Sigma \subset M$, 
but not in $\Sigma$, which will lie in the event horizon. In case $\bar{\Sigma}$ can be compactified by adding a point at infinity, $\bar{\Sigma} \cup\{\infty\}$ can be employed instead of a submanifold $\Sigma^{\prime}$.

The basic idea of our proof is to show that, for any $\Sigma^{\prime}$ as above, $H_{1}\left(\Sigma^{\prime}\right)$ must be finite, because if $H_{1}\left(\Sigma^{\prime}\right)$ is infinite, one can "sew" a timelike curve from $\mathcal{I}^{-}$to $\mathcal{I}^{+}$through $\mathcal{C}_{K}$ in such a way that it is necessarily linked and not deformable to infinity. This would violate the topological censorship theorem, so we conclude that $H_{1}\left(\Sigma^{\prime}\right)$ is finite. Now finiteness of $H_{1}\left(\Sigma^{\prime}\right)$ is a weaker restriction on the topology than is simply connectedness. Nevertheless, it turns out that the finiteness of $H_{1}\left(\Sigma^{\prime}\right)$ is enough of a restriction on $\Sigma^{\prime}$ to imply, as long as $\Sigma^{\prime}$ or $\partial \Sigma^{\prime}$ is orientable, that $\partial \Sigma^{\prime}$ must be a disjoint union of 2 -spheres [7]. We will argue below that $\partial \Sigma^{\prime} / \Sigma$ is always a subset of the event horizon. If in fact it consists of closed cross sections of components of the horizon, it then follows that these components are 2-spheres.

In summary, the theorem to be proved is this:

Theorem: Let $M$ be a four dimensional asymptotically flat spacetime satisfying the averaged null energy condition, let $K$ be a cut of $\mathcal{I}^{-}$, and suppose that the domain of outer communication $\mathcal{C}_{K}:=I^{+}[K] \cap I^{-}\left[\mathcal{I}^{+}\right]$to the future of $K$ is globally hyperbolic. Suppose further that the closure of a Cauchy surface $\Sigma$ for $C_{K}$ is a topological 3-manifold with compact boundary $\partial \Sigma$, and that $\Sigma^{\prime}$ is a compact submanifold of $\bar{\Sigma}$ with a single 2-spherical boundary $S$ in $\Sigma$ and possibly other boundary components in $M / \Sigma$. Then

(1) $H_{1}\left(\Sigma^{\prime}\right)$ is finite;

(2) Either $\partial \Sigma^{\prime}$ consists of a disjoint union of 2-spheres, or $\Sigma^{\prime}$ is nonorientable and $\partial \Sigma^{\prime}$ contains a projective plane; and

(3) $\partial \Sigma=\partial I^{+}[K] \cap \partial I^{-}\left[\mathcal{I}^{+}\right]$so, in particular, $\partial \Sigma$ is a subset of the event horizon.

Before presenting the proof of this theorem, let us offer some fuzzy intuitive remarks about what it is that will have been proved. Suppose the topology of a one-component event horizon of a black hole is unchanging to the future of some slice. Then it seems that generically there will exist a cut $K$ of $\mathcal{I}^{-}$such that $\partial I^{+}[K]$ will intersect the horizon transversly in a closed 
surface forming a cross section. Our result then implies that this cross section must be a 2-sphere. More generally, it seems plausible that if the topology of the horizon is merely unchanging for "sufficiently long", say, much longer than the light crossing time corresponding to the asymptotic mass of that component, then there will exist such a cut $K$. If the horizon has more than one component, then one expects that similar statements would apply. Further, if a given component is sufficiently well separated from the other components so that it is surrounded by a fairly flat region, then one would expect the other components to be irrelevant.

The proof involves the concept of a properly embedded, two-sided, nonseparating surface $D[7]$. A properly embedded surface $D$ is a compact, connected 2-submanifold of a 3-manifold $\Sigma$ with $\partial D=D \cap \partial \Sigma$. It is helpful to visualize the situation using the simple example of $R^{3}$ minus a solid torus in which case a disk in $\Sigma$ whose boundary lies on the inner cycle of the torus serves as the surface $D$. This, in fact, is the example we generalized to come up with the strategy of this proof. A more subtle example is provided by $R^{3}$ minus a solid knotted torus, in which case $D$ can be a Seifert surface [8] bounded by a knotted cycle on the torus. An example where $D$ has no boundary is given by a wormhole $S^{1} \times S^{2}$ minus a point, in which case $D$ could be an $S^{2}$ cross section of the wormhole.

We now present our proof that $H_{1}\left(\Sigma^{\prime}\right)$ must be finite. Suppose on the contrary that $H_{1}\left(\Sigma^{\prime}\right)$ is infinite. Then $\Sigma^{\prime}$ contains a two-sided, properly embedded, nonseparating surface $D[7]$. Without loss of generality we can assume that $D$ does not intersect the spherical boundary $S$, since this boundary can be eliminated by a one-point compactification. Thus $D$ is properly embedded, two-sided, and nonseparating in $\bar{\Sigma}$ as well. Because $D$ is two-sided, one can define an intersection number of a closed curve $\alpha$ with $D$ as the total number of intersections of $\alpha$ with $D$, each intersection counted as \pm 1 according as $\alpha$ passes from $D^{-}$to $D^{+}$or vice versa, where $D^{ \pm}$are the two sides of $D$. Because $D$ is a properly embedded surface, the intersection number of $\alpha$ with $D$ is a topological invariant. If $\alpha$ has nonzero intersection number with $D$, then $\alpha$ is "linked" and cannot be contracted to a point or deformed to infinity. The nonseparating property of $D$ will be invoked below.

Let $\Sigma$ now denote one particular Cauchy surface in the foliation of $\mathcal{C}_{K}$, let $D$ be a two-sided, nonseparating, properly embedded surface in $\Sigma$, and let $\tilde{D}$ denote the flow of $D$ along the orbits of $t^{a}$. We adopt the name "C-curves" for timelike curves from $\mathcal{I}^{-}$to $\mathcal{I}^{+}$in $\mathcal{C}_{K}$. Define the subset $F_{n} \subset \mathcal{C}_{K} / \tilde{D}$ as the 
collection of all points in $\mathcal{C}_{K} / \tilde{D}$ through which there exists a C-curve whose future half has intersection number $n$ with $\tilde{D}$. (Equivalently, the projection of the future half curve into $\Sigma$ along the orbits of $t^{a}$ has intersection number $n$ with $D$.) Note that since $\mathcal{C}_{K}$ is the product $\Sigma \times R$, the intersection number of a C-curve with $\tilde{D}$ is well defined and is a topological invariant of C-curves.

It is easy to see that each $F_{n}$ is an open set. Furthermore, since any point of $\mathcal{C}_{K}$ is in $I^{-}\left[\mathcal{I}^{+}\right]$, we have $\mathcal{C}_{K} / \tilde{D}=\cup_{n} F_{n} . F_{0}$ is clearly nonempty, since there are points near spatial infinity through which there must exist C-curves that do not meet $\tilde{D}$. At least one of the other $F_{n}$ 's must also be nonempty. To see why, consider any point $d$ in $\tilde{D}$. There must exist a C-curve $\delta$ through $d$ which intersects $\tilde{D}$ transversely at $d$. If the half of $\delta$ to the future of $d$ has vanishing intersection number, then $p$ just to the past of $d$ on $\delta$ will be in $F_{1}$. If the half of $\delta$ to the future of $d$ has nonvanishing intersection number $m$, then $p$ just to the future of $d$ on $\delta$ will be in $F_{m}$.

Since $F_{0}$ and at least one of the other $F_{n}$ 's are nonempty, we have shown that $\mathcal{C}_{K} / \tilde{D}$ can be written as a union of two nonempty open sets, $\mathcal{C}_{K} / \tilde{D}=F_{0} \cup\left(\cup_{n \neq 0} F_{n}\right)$. Since $\mathcal{I}^{-}$is connected it follows that $\Sigma$ is connected, and removing the nonseparating surface $D$ cannot change this, so $\Sigma / D$ is connected and therefore so is $\mathcal{C}_{K} / \tilde{D}=(\Sigma / D) \times R$. Thus the two open sets cannot be disjoint, so there must be a point $p$ in both $F_{0}$ and $F_{m}$ for some nonzero $m$.

Topological censorship implies that all C-curves through $p$ are unlinked with $\tilde{D}$, so that the future and past halves of all C-curves through $p$ must have opposite intersection numbers. However, since $p$ is in both $F_{0}$ and $F_{m}$, we can therefore find a C-curve through $p$ whose past half has zero intersection number and whose future half has intersection number $m$. But this curve has a net nonzero intersection number $m$, and is therefore linked, which yields a contradiction. We thus conclude that no surface such as $D$ can exist, and therefore that $H_{1}\left(\Sigma^{\prime}\right)$ must be finite for any compact submanifold $\Sigma^{\prime} \subset \bar{\Sigma}$ with 2-sphere boundary in $\Sigma$.

Finiteness of $H_{1}\left(\Sigma^{\prime}\right)$ implies that either $\partial \Sigma^{\prime}$ consists of a disjoint union of 2-spheres, or $\Sigma^{\prime}$ is nonorientable and $\partial \Sigma^{\prime}$ contains a projective plane[7]. This establishes the second claim of the theorem.

Finally, how is $\partial \Sigma$ related to the horizon? We now argue that $\partial \Sigma=$ $\partial I^{+}[K] \cap \partial I^{-}\left[\mathcal{I}^{+}\right]$, the third claim of the theorem. Any point $x$ in $\partial \Sigma$ is also in $\partial \mathcal{C}_{K}=\partial\left(I^{+}[K] \cap I^{-}\left[\mathcal{I}^{+}\right]\right)$and, since $\Sigma$ is a Cauchy surface for $\mathcal{C}_{K}$, $x$ cannot be in the interior of either $I^{+}[K]$ or $I^{-}\left[\mathcal{I}^{+}\right]$. Therefore, $x$ must lie 
in $\partial I^{+}[K] \cap \partial I^{-}\left[\mathcal{I}^{+}\right]$. Conversely, if $x$ is in $\partial I^{+}[K] \cap \partial I^{-}\left[\mathcal{I}^{+}\right] \subset \partial \mathcal{C}_{K}$, then $x$ must be in the closure of $\Sigma$. Otherwise, there would exist an open set around $x$ which does not intersect $\Sigma$, and one could therefore find a timlike curve through a point in $\mathcal{C}_{K}$ that does not intersect the Cauchy surface $\Sigma$. Furthermore, $x$ could not be an interior point of $\Sigma$, since $x \in \partial \mathcal{C}_{K}$. Thus $x \in \partial \Sigma$. This completes the proof of our theorem.

The above argument shows that $\partial \Sigma$ is the subset $\partial I^{+}[K] \cap \partial I^{-}\left[\mathcal{I}^{+}\right]$of the event horizon. However, we do not know that it is a union of cross sections of components of the horizon. With the additional hypothesis that no generator of $I^{+}[K]$ becomes a horizon generator we can prove that $\partial I^{+}[K] \cap \partial I^{-}\left[\mathcal{I}^{+}\right]$ intersects no horizon generator more than once. If some generator is intersected in two points $p$ and $q$, then the later of the two points $q$ will be joined to $K$ by a segment of the horizon generator back to $p$ followed by a generator of $\partial I^{+}[K]$ back to $K$. If no generator of $I^{+}[K]$ becomes a horizon generator then these two curves must form a broken future pointing null geodesic, which would contradict the fact that $q$ is in the boundary $\partial I^{+}[K]$.

The remainder of this paper consists of several remarks.

i) We have not proved anything about $H_{1}(\Sigma)$ nor $\pi_{1}(\Sigma)$ in more than three spatial dimensions. But, even if we could establish some such result, it would say absolutely nothing about the topology of the horizon by itself. The reason is that, in more than three dimensions, any orientable manifold that is the boundary of some other manifold is also the boundary of a simply connected manifold. This is because one can always kill the homotopy classes by surgery without changing the boundary of the manifold [9]. [To kill the homotopy class of a curve $\lambda$ in an $n$-manifold, "thicken" $\lambda$ to obtain a solid tube $S^{1} \times D^{n-1}$, cut out the solid tube, and replace the solid tube by the simply connected manifold $D^{2} \times S^{n-2}$ (which has the same boundary, $S^{1} \times$ $S^{n-2}$, as the solid tube.)] For example $S^{1} \times D^{3}$ is nonsimply connected with nonsimply connected boundary $S^{1} \times S^{2}$. Surgery yields the simply connected manifold $D^{2} \times S^{2}$ with the same boundary.

ii) A $3+1$ dimensional static black hole with cylindrical event horizon has recently been found in the presence of a negative cosmological constant [10]. This spacetime does not contradict our result because it is not asymptotically flat in the usual sense, and there is no simply connected neighborhood of infinity. However, although the proof of topological censorship in 
[4] employed the existence of a simply connected neighborhood of infinity, it appears likely that the theorem can be extended to spacetimes with no such neighborhood. [The spacetime of [10] is asymptotically anti-de Sitter in the "transverse" directions rather than asymptotically flat, so it is not immediately clear whether or not the topological censorship theorem would apply to it anyway. Nevertheless, we believe it is not hard to extend the theorem to cover this asymptotic structure.] If this is the case, topological censorship would still imply that all causal curves from $\mathcal{I}^{-}$to $\mathcal{I}^{+}$are deformable to a neighborhood of infinity. Nevertheless, the presence of a cylindrical horizon does not prevent this from being true. Any curve that wraps around the horizon can just be pulled out to wrap around infinity. Similar comments apply to the recently discussed $2+1$ dimensional black holes with circular event horizons [11.

iii) A 3+1 dimensional static black hole with toroidal event horizon can be obtained by taking the product of a static $1+1$ dimensional black hole with a flat $T^{2}$. This spacetime does not contradict either Hawking's theorem or ours, for several independent reasons. The $1+1$ spacetime has positive curvature and the transverse space is flat, so one finds that the effective $4 \mathrm{~d}$ stress tensor has only negative transverse pressure components. Thus all energy conditions are violated [12]. Furthermore, this spacetime is not asymptotically flat in the usual sense. The expansion of a null congruence orthogonal to a spacelike cut of null infinity can vanish in this spacetime, so one can not even argue that a marginally trapped surface must lie inside an event horizon. Moreover, there is no simply connected neighborhood of infinity. The last two problems can be circumvented by splicing on to a standard asymptotically flat metric outside some radius [13]. Evidently, however, this cannot be done without violating the averaged null energy condition, since otherwise one would have a marginally trapped surface visible from $\mathcal{I}^{+}$.

$i v$ ) The recent work of Hughes et. al. [14 demonstrates numerically that a spinning toroid of collisionless matter can collapse to form a black hole whose event horizon is initially toroidal and later becomes spherical. The existence of the temporarily toroidal horizon does not obviously contradict our theorem since the toroidal topology is not unchanging to the future of some slice. However, our theorem implies that whenever a temporarily nonspherical horizon occurs, it must not remain nonspherical long enough to permit the existence of a cut $K$ of $\mathcal{I}^{-}$such that the boundary $\partial \Sigma$ of the Cauchy surface for $\mathcal{C}_{K}$ is a non-spherical cross section. (This fact seems closely related 
to the puzzle raised in remark (vi) below.) Evidently, for any cut $K$, either $\partial \Sigma$ is a spherical cross section to the future of the torus, or it is spherical but fails to be a cross section, or $\Sigma \cup \partial \Sigma$ fails to be a 3-manifold with compact boundary $\partial \Sigma$.

$v$ ) The topological censorship theorem proved in [4] showed that every causal curve from $\mathcal{I}^{-}$to $\mathcal{I}^{+}$must be deformable to lie in a simply connected neighborhood of infinity. We conjecture that the theorem may be strengthened to show, with the same assumptions, that each such causal curve must be deformable to infinity through a family of causal curves that all emerge from the same point of $\mathcal{I}^{-}$. We will call such deformations "causal". To establish this conjecture, it would seem natural to adapt the alternate proof of the topological censorship theorem, mentioned in [1], which uses the concept of "fastest curve" from a given generator of $\mathcal{I}^{-}$to a given generator of $\mathcal{I}^{+}$.

vi) Our conjecture in the preceding remark poses the following puzzle regarding the temporarily toroidal horizon of Ref. [14]. Consider the one parameter family of null rays that travel along the axis of symmetry, in particular the rays that arrive at the location of the spinning toroid at intermediate times, after the toroidal horizon has formed but before it has become spherical. It seems at first that some of these rays will make it back out to infinity but will not be causally deformable to infinity, because the required deformation would necessarily intersect the horizon. Thus either our conjectured strengthening of the topological censorship theorem is wrong, or in fact all these null rays are causally deformable to infinity.

Since the toroidal horizon persists only for a time of order $M$, a time advance of order $M$ would suffice to disentangle the ray from the horizon. Perhaps by increasing the impact parameter, i.e., moving off axis but still coming in from the same point of $\mathcal{I}^{-}$, a causal curve could arrive at the spinning toroid before the formation of the event horizon, and could thus be further causally deformed to infinity.

We are grateful to D. Brill, S. Corley, W. Goldman, C. W. Misner, J. Schafer, and J. Z. Simon for helpful discussions, and especially grateful to G. Galloway, P. Chruściel, and R.M. Wald for comments and corrections on a draft of this paper. T. J. would like to thank the Institute for Theoretical Physics at the University of Bern for hospitality and Tomalla Foundation Zurich for support while this work was being completed. This work was 
supported in part by NSF Grant PHY91-12240.

\section{References}

[1] Hawking, S. W., Comm. Math. Phys 25, 152 (1972); Hawking, S. W. and Ellis, G. F. R., The Large Scale Sructure of Spacetime, Cambridge University Press (Cambridge, 1973).

[2] Gannon, D., Gen. Rel. Grav. 7, 219 (1976).

[3] Browdy, S. and Galloway, G.J., preprint; Galloway, G., "Least area tori, black holes and topological censorship", to appear in Contemp. Math. 170, eds. J. Beem and K.L. Duggal (Amer. Math. Soc., Providence, 1994)

[4] Friedman, J. L., Schleich, K. and Witt, D. M., Phys. Rev. Lett. 71, 1486 (1993).

[5] Chruściel, P.T. and Wald, R.M., "On the topology of stationary black holes", preprint (1994).

[6] Wald, R. M., General Relativity, The University of Chicago Press (Chicago, 1984).

[7] Hempel, J., 3-Manifolds, Princeton University Press (Princeton, 1976).

[8] Kauffman, L. H., On Knots, Princeton University Press (Princeton, 1987).

[9] Milnor, J., in Proceedings of Symposia in Pure Mathematics, volume III: Differential Geometry, American Mathematical Society (Providence, 1961).

[10] Lemos, J. P. S., gr-qc/9404041.

[11] Bañados, M., Teitelboim, C., and Zanelli, J., Phys. Rev. Lett 69, 1849 (1992). 
[12] Horowitz, G. T., in String theory and quantum gravity '92, ed. J. Harvey et. al., World Scientific (Singapore, 1993).

[13] Geroch, R., and Hartle, J., J. Math. Phys. 23, 680 (1982).

[14] Hughes, S. A., et. al., Phys. Rev. D 49, 4004 (1994). 